\title{
Psychometric properties of the Fear of COVID-19 Scale: A response to de Medeiros et al. "Psychometric properties of the Brazilian version of the Fear of COVID-19 Scale (FCV-19S)"
}

\author{
Chung-Ying Lin ${ }^{1,2,3,4} \cdot$ Mark D. Griffiths ${ }^{5} \cdot$ Amir H. Pakpour $^{6}$ \\ Accepted: 29 December 2021 \\ ๑) The Author(s), under exclusive licence to Springer Science+Business Media, LLC, part of Springer Nature 2022
}

\section{Dear editors,}

de Medeiros et al. (2021) recently published a study in your journal examining how Brazilian version of the Fear of COVID-19 Scale (FCV-19S) performed in the Portuguese language among Brazilians. The authors used different psychometric testing methods, including exploratory factor analysis, item response theory, and confirmatory factor analysis to indicate the robustness of the FCV-19S's psychometric properties. They confirmed that the use of FCV-19S was appropriate and recommended its use among Brazilians during the COVID-19 pandemic. As the original co-developers of FCV-19S, we welcome all empirical evidence concerning the FCV-19S because the accumulated psychometric knowledge of FCV-19S will be of benefit to the scientific community. However, we have some major concerns with their study.

First, the FCV-19S can be used freely by any researchers, treatment providers and healthcare practitioners, as well as

Amir H. Pakpour

Amir.pakpour@ju.se

1 Institute of Allied Health Sciences, College of Medicine, National Cheng Kung University, Tainan, Taiwan

2 Department of Public Health, College of Medicine, National Cheng Kung University, Tainan, Taiwan

3 Department of Occupational Therapy, College of Medicine, National Cheng Kung University, Tainan, Taiwan

4 Biostatistics Consulting Center, College of Medicine, National Cheng Kung University Hospital, National Cheng Kung University, Tainan, Taiwan

5 International Gaming Research Unit, Psychology Department, Nottingham Trent University, 50 Shakespeare Street, Nottingham NG1 4FQ, UK

6 Department of Nursing, School of Health and Welfare, Jönköping University, Barnarpsgatan 39, 55111 Jönköping, Sweden laypeople. However, we consider that removing scale items without informing or consulting the developers is poor scientific etiquette and may jeopardize the latent concept of fear of COVID-19 in the scale we co-developed (see: Ahorsu et al., 2020; Lin et al., 2021). More specifically, we used rigorous methodology to develop the FCV-19S with full consideration of the fear concept (using 30 different validated 'fear' scales as a basis for developing the instrument). However, de Medeiros et al. (2021) did not inform and request opinions from us as the developers of FCV-19S before they developed a brief version of FCV-19S. We believe that they should have to made a formal request to us as the original developers of the instrument before independently removing items and shortening it. Personally, we feel this is somewhat disrespectful.

Second, based on the results reported by de Medeiros et al. (2021), it is obvious that the seven-item FCV-19S (i.e., our original FCV-19S) does not perform any worse than the shortened four-item FCV-19S. More specifically, the factor loadings derived from exploratory factor analysis were between 0.73 and 0.88 for the original FCV-19S, and between 0.58 and 0.80 for the brief FCV-19S. The factor loadings derived from confirmatory factor analysis were between 0.47 and 0.81 for the original FCV-19S, and between 0.63 and 0.82 for the brief FCV-19S. The fit indices were all satisfactory for the original FCV-19S (comparative fit index $[\mathrm{CFI}]=0.985$; Tucker-Lewis index $[\mathrm{TLI}]=0.977$; and root mean square error of approximation $[$ RMSEA] $=0.073$ ) as they were for the brief FCV-19S $(\mathrm{CFI}=0.996$; $\mathrm{TLI}=0.989$; RMSEA $=0.039)$. Moreover, both the original FCV-19S and brief FCV-19S had good internal consistency in regards to Cronbach's alpha and McDonald's omega (alpha $=0.87$ for both original and brief FCV-19S; omega $=0.86$ for original FCV-19S and 0.83 for brief FCV-19S). The psychometric information was not that different between the original FCV-19S (theta $=6.33$ to 4.00 ) and the brief FCV-19S (theta $=6.99$ to 4.09 ). In 
sum, the psychometric properties are similar between our original FCV-19S and the brief FCV-19S in the results of de Medeiros et al. (2021). Additionally, the findings reported by de Medeiros et al. (2021) are comparable to other studies testing the original FCV-19S (e.g., Huarcaya-Victoria et al., 2020; Lin et al., 2021; Martínez-Lorca et al., 2020).

Third, the major benefit of using a brief instrument is to save time in a busy setting ( $\mathrm{Li}$ et al., 2021), especially when the participants have a condition that burdens them when completing the instrument (e.g., participants being very old). However, we believe that our seven-item FCV-19S with easy-to-understand item descriptions do not take too much time when any individual uses it. Indeed, it only takes a couple of minutes at most to complete the original sevenitem FCV-19S. Developing an even shorter version of the FCV-19S does not appear to have any significantly enhanced benefits by slightly reducing the time taken to complete the FCV-19S and provides less useful information overall. In conclusion, we strongly recommend that anyone, including academic researchers, healthcare practitioners, and treatment providers, should think twice before using the brief version of our scale.

Funding The authors did not receive support from any organization for the submitted work.

Data Availability Data sharing not applicable to this article as no datasets were generated or analysed during the current study.

\section{Declarations}

Ethical Approval All the procedures performed in studies involving human participants were in accordance with the ethical standards of the Ono Academic College institutional research board and with the 1964 Helsinki declaration and its later amendments or comparable ethical standards.
Conflict of Interests The authors declared no potential conflicts of interest with respect to the research, authorship, and/or publication of this article.

\section{References}

Ahorsu, D. K., Lin, C. Y., Imani, V., Saffari, M., Griffiths, M. D., \& Pakpour, A. H. (2020). The Fear of COVID-19 Scale: Development and initial validation. International Journal of Mental Health and Addiction. Advance online publication. https://doi. org/10.1007/s11469-020-00270-8

de Medeiros, E. D., Reis, L. M., Guimarães, C., da Silva, P., Monteiro, R. P., Coelho, G., Guimarães, C., Martins, E., \& de França, L. (2021). Psychometric properties of the Brazilian version of the Fear of COVID-19 Scale (FCV-19S). Current Psychology, 1-10. Advance online publication. https://doi.org/10.1007/ s12144-021-01476-2

Huarcaya-Victoria, J., Villarreal-Zegarra, D., Podestà, A., \& LunaCuadros, M. A. (2020). Psychometric properties of a Spanish version of the Fear of COVID-19 Scale in general population of Lima, Peru. International Journal of Mental Health and Addictio. Advance online publication. https://doi.org/10.1007/ s11469-020-00354-5

Li, Y. P., Lin, C. Y., Hu, F. W., \& Shih, S. A. (2021). Short versions of the Geriatric Depression Scale (GDS) among widowed older people in Taiwan: Comparing their psychometric properties. Australasian Journal on Ageing, https://doi.org/10.1111/ajag.12942. Advance online publication. https://doi.org/10.1111/ajag.12942

Lin, C. Y., Hou, W. L., Mamun, M. A., Aparecido da Silva, J., Broche-Pérez, Y., Ullah, I., ... \& Pakpour, A. H. (2021). Fear of COVID-19 Scale (FCV-19S) across countries: Measurement invariance issues. Nursing Open, 8(4), 1892-1908. https://doi. org/10.1002/nop2.855

Martínez-Lorca, M., Martínez-Lorca, A., Criado-Álvarez, J. J., \& Armesilla, M. D. C. (2020). The Fear of COVID-19 Scale: Validation in Spanish university students. Psychiatry Research, 293, 113350. https://doi.org/10.1016/j.psychres.2020.113350

Publisher's Note Springer Nature remains neutral with regard to jurisdictional claims in published maps and institutional affiliations. 